\title{
Transformation or Progression from Adenocarcinoma to Small Cell Lung Cancer Detected by Serially Tracking Mutations in the Blood
}

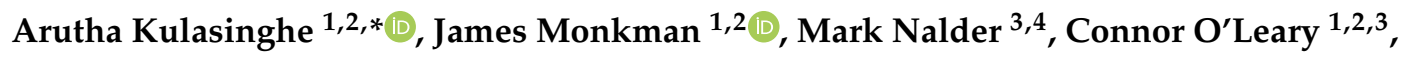 \\ Rahul Ladwa ${ }^{3,4}$ and Ken O'Byrne ${ }^{1,2,3}$ \\ 1 The School of Biomedical Sciences, Institute of Health and Biomedical Innovation, Queensland University of \\ Technology, 37 Kent Street, Woolloongabba, QLD 4102, Australia; james.monkman@qut.edu.au (J.M.); \\ connor.oleary@health.qld.gov.au (C.O.); k.obyrne@qut.edu.au (K.O.) \\ 2 Translational Research Institute, Brisbane, QLD 4102, Australia \\ 3 Princess Alexandra Hospital, Brisbane, QLD 4102, Australia; Mark.Nalder@health.qld.gov.au (M.N.); \\ rahul.ladwa@health.qld.gov.au (R.L.) \\ 4 University of Queensland, Brisbane, QLD 4102, Australia \\ * $\quad$ Correspondence: Arutha.kulasinghe@qut.edu.au; Tel.: +61-7-3138-6227
}

Received: 10 October 2020; Accepted: 3 November 2020; Published: 4 November 2020

\begin{abstract}
Background: Circulating tumour DNA (ctDNA) has emerged as a promising biomarker for monitoring non-small-cell lung cancer (NSCLC). This has enabled the monitoring of clinically actionable mutations over the course of therapy. Case presentation: We present the case of a 74-year-old female who was treated with EGFR inhibitors for NSCLC which later transformed to SCLC. The kinetics of ctDNA was monitored by measuring the EGFR Exon 19 mutant L747-A750 $>\mathrm{P}$ and PIK3CA E545K over the course of therapy. Stabilisation of the PIK3CA mutation was found in response to therapy, however there appeared to be increasing levels of the EGFR mutant, potentially reflective of untreated EGFR-driven disease. Conclusion: The key finding described here, revealed by mutational tracking of mutations from the blood, is that clinically actionable mutations are assessable and may demonstrate clinical utility in measuring disease burden, multiple clones and progression non-invasively for lung cancer.
\end{abstract}

Keywords: circulating tumour dna; non-small-cell lung cancer; mutations

\section{Introduction}

There have been studies in non-small-cell lung cancer (NSCLC) with mutated EGFR which returns as SCLC when there is resistance to tyrosine kinase inhibitors and reports about the co-existence of NSCLC and SCLC [1]. Whilst repeat tissue biopsy can potentially identify these changes, it remains a limited and invasive procedure to perform, which does not reflect dynamic tumoural changes in real time. Herein, we assessed the changes in circulating tumour DNA (ctDNA) in EGFR-mutated NSCLC over the course of treatment using the Lung UltraSeek mutation Panel (Agena Biosciences, San Diego, CA, USA) [2].

\section{Case Report}

We report a case of a 74-year-old female who proceeded to a right lower lobectomy in for a malignant pulmonary lesion. She had no smoking history. Histopathology revealed a $29 \mathrm{~mm}$, acinar predominant adenocarcinoma with malignant cells involved in one hilar lymph node (stage IIb-AJCC 8th edition). Competitive allele-specific TaqMan PCR detected a deletion in exon 19 of the EGFR gene. Immunohistochemistry for Anaplastic Lymphoma Kinase was negative using the D5F3 antibody. 
She received one adjuvant cycle of cisplatin and vinorelbine, but further cycles were cancelled due to poor tolerance.

She developed recurrence in a right subcarinal lymph node 22 months later. Repeat biopsy confirmed adenocarcinoma, however no further immunohistochemistry was performed. She was commenced on epidermal growth factor inhibitor Erlotinib (Figure 1; T0-T1) which continued for six months when she developed an enlarging right hilar lymph node. Serial blood samples were taken from the patient and EGFR and PIK3CA mutations monitored via liquid biopsy. Repeat biopsy of the hilar node revealed a highly cellular specimen with strongly positive immunostaining for CK8/18, CD56 and synaptophysin with a Ki67 of at least 75\% consistent with transformation to small cell neuroendocrine carcinoma. Next-generation sequencing using the TruSight Tumour panel confirmed persistent exon 19 EGFR deletion c.2239_2248delinsC p.(Leu747_Ala750delinsPro). Thr790Met exon 20 EGFR missense mutation was not detected. She was initially not suitable for systemic treatment but functionally improved despite progression of her disease and completed four cycles of chemotherapy with Carboplatin and Etoposide with significant partial response to treatment (Figure 1; T1-T2; increasing EGFR_pL747_A750toP and PIK3CA_pE545K). She developed progressive disease and completed four further cycles of Carboplatin and Etoposide but unfortunately developed rapid disease progression. She was commenced on chemotherapy with Cyclophosphamide, Doxorubicin and Vincristine (CAV) (Figure 1; T2-T3) and completed four cycles with stable radiological disease. This was reflective in the blood by stabilisation of the PIK3CA_pE545K mutation. Her disease remained stable when she once again developed progressive disease (Figure 1; T3). Between T2 and T3, the EGFR_pL747_A750toP continued to increase, potentially reflective of untreated EGFR-driven disease. She received once cycle of third-line chemotherapy with Irinotecan, but her health deteriorated rapidly, and she died a month later.

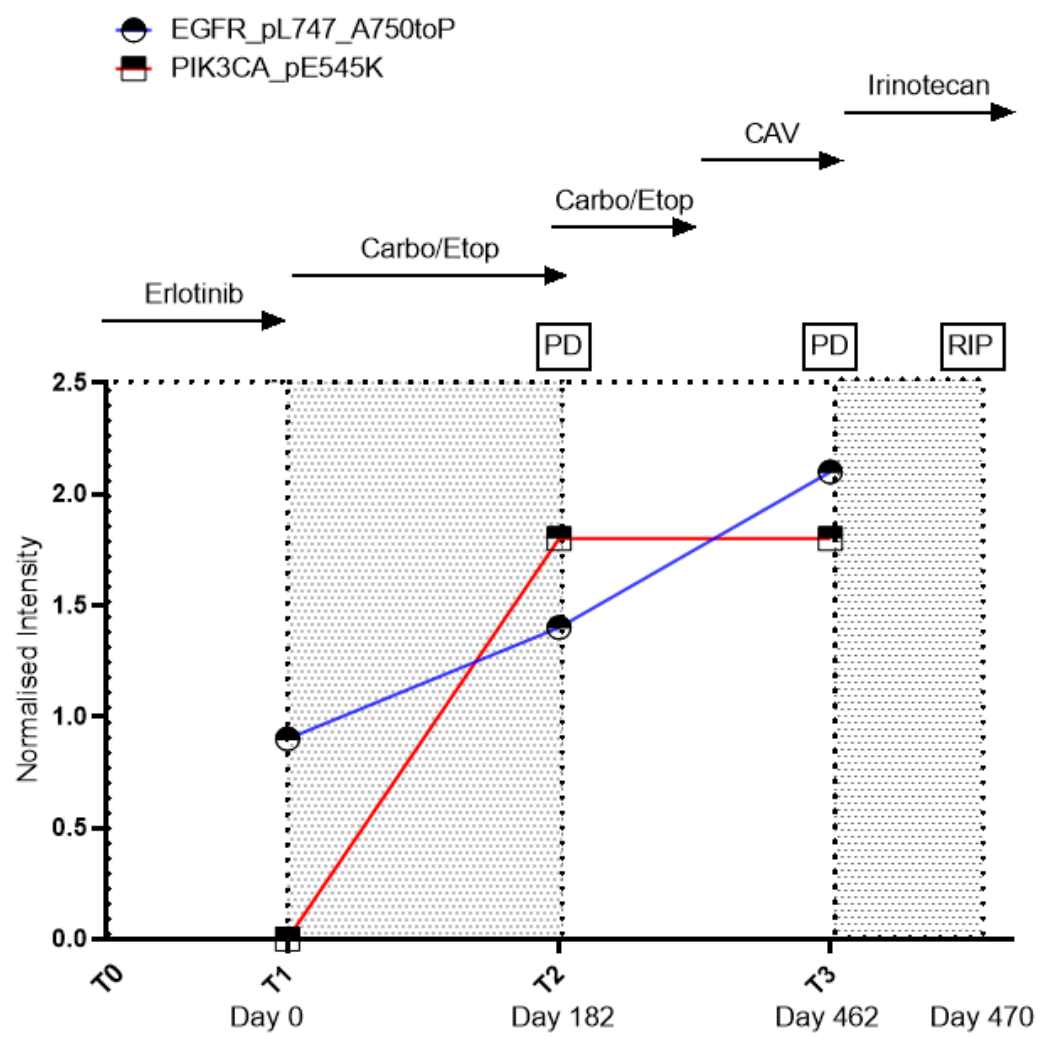

Figure 1. Longitudinal measurements of circulating tumour DNA (ctDNA) by Ultraseek in the patient. The tracking of EGFR exon 19 deletion (EGFR_pL747_A750toP) is shown in blue and PIK3C mutation (PIK3CA_E545K) is shown in red. Disease status by radiological imaging is indicated in the boxes: PD (progressive disease); RIP (death). 
The key finding described here, revealed by mutational tracking of mutations from the blood, is that clinically actionable mutations are assessable and may demonstrate clinical utility in measuring disease burden, multiple clones and progression non-invasively. Moreover, the L747-A750 > P mutation may exhibit limited sensitivity to first-generation tyrosine kinase inhibitor TKI Elortinib [3]. Widespread use of mutational profiling of the blood in NSCLC may assist in understanding the molecular biology of the disease.

\section{Ethics Approvals}

This study was conducted at the Princess Alexandra Hospital in Brisbane. Ethics approval was obtained from the Metro South Health District Human Research Ethics Committee in accordance with the National Health and Medical Research Councils guidelines (HREC/11/QPAH/331). All methodologies were performed in accordance with these ethical guidelines and regulations. This study has institutional approval from the Queensland University of Technology human ethics committee (1100001420). Informed consent was obtained from the patient.

Funding: This study was funded by Translational Research Institute Spore grant. A.K. is supported by NHMRC ECF Fellowship (APP1157741). The Translational Research Institute receives funding from the Australian government. The authors are grateful for the support from Darryl Irwin (Agena Biosciences Brisbane) and the clinical trials support at the Princess Alexandra Hospital for their assistance.

Conflicts of Interest: The authors declare no conflict of interest.

\section{References}

1. Yu, H.A.; Arcila, M.E.; Rekhtman, N.; Sima, C.S.; Zakowski, M.F.; Pao, W.; Kris, M.G.; Miller, V.A.; Ladanyi, M.; Riely, G.J. Analysis of tumor specimens at the time of acquired resistance to EGFR-TKI therapy in 155 patients with EGFR-mutant lung cancers. Clin. Cancer Res. 2013, 19, 2240-2247. [CrossRef]

2. Smyth, R.J.; Toomey, S.M.; Sartori, A.; O'Hanrahan, E.; Cuffe, S.D.; Breathnach, O.S.; Morgan, R.K.; Hennessy, B.T. Brief Report on the Detection of the EGFR T790M Mutation in Exhaled Breath Condensate from Lung Cancer Patients. J. Thorac. Oncol. 2018, 13, 1213-1216. [CrossRef]

3. Truini, A.; Starrett, J.H.; Stewart, T.; Ashtekar, K.; Walther, Z.; Wurtz, A.; Lu, D.; Park, J.H.; DeVeaux, M.; Song, X.; et al. The EGFR Exon 19 Mutant L747-A750 > P Exhibits Distinct Sensitivity to Tyrosine Kinase Inhibitors in Lung Adenocarcinoma. Clin. Cancer Res. 2019, 25, 6382-6391. [CrossRef]

Publisher's Note: MDPI stays neutral with regard to jurisdictional claims in published maps and institutional affiliations.

(C) 2020 by the authors. Licensee MDPI, Basel, Switzerland. This article is an open access article distributed under the terms and conditions of the Creative Commons Attribution (CC BY) license (http://creativecommons.org/licenses/by/4.0/). 